\title{
Elementary Statistical Models for Vector Collision-Sequence Interference Effects with Poisson-Distributed Collision Times
}

\author{
John Courtenay Lewis \\ Department of Physics and Physical Oceanography, Memorial University of Newfoundland, St. John's, NL, Canada A1B 3X7 \\ Correspondence should be addressed to John Courtenay Lewis, court@physics.mun.ca
}

Received 22 April 2009; Accepted 22 May 2009

Academic Editor: Roland Stamm

Copyright (C 2010 John Courtenay Lewis. This is an open access article distributed under the Creative Commons Attribution License, which permits unrestricted use, distribution, and reproduction in any medium, provided the original work is properly cited.

\begin{abstract}
In a recent paper (Lewis, 2008) a class of models suitable for application to collision-sequence interference was introduced. In these models velocities are assumed to be completely randomized in each collision. The distribution of velocities was assumed to be Gaussian. The integrated induced dipole moment $\mu_{k}$, for vector interference, or the scalar modulation $\mu_{k}$, for scalar interference, was assumed to be a function of the impulse (integrated force) $\mathbf{f}_{k}$, or its magnitude $f_{k}$, experienced by the molecule in a collision. For most of (Lewis, 2008) it was assumed that $\mu_{k} \propto \mathbf{f}_{k}$ and $\mu_{k} \propto f_{k}$, but it proved to be possible to extend the models, so that the magnitude of the induced dipole moment is equal to an arbitrary power or sum of powers of the intermolecular force. This allows estimates of the infilling of the interference dip by the disproportionality of the induced dipole moment and force. One particular such model, using data from (Herman and Lewis, 2006), leads to the most realistic estimate for the infilling of the vector interference dip yet obtained. In (Lewis, 2008) the drastic assumption was made that collision times occurred at equal intervals. In the present paper that assumption is removed: the collision times are taken to form a Poisson process. This is much more realistic than the equal-intervals assumption. The interference dip is found to be a Lorentzian in this model.
\end{abstract}

\section{Introduction}

Spectra resulting from dipole moments induced in molecular collisions typically have the form of broad bands with widths determined by the durations of those collisions [1]. However, these broad bands often exhibit narrow features, which result from the coherence or correlation of induced dipole moments extending over successive or sometimes many collisions. The most conspicuous such features are the vector intercollisional interference dips found in the fundamental bands of $\mathrm{H}_{2}-\mathrm{X}$ spectra and in the pure translational bands of mixtures of rare gas atoms [2]. Also well known are scalar collisional interference features found in the $R$ and $P$ transitions in the fundamental bands of HD - X spectra [35 ] and the corresponding $R$ transitions in the pure rotational spectra $[6,7]$. The terms "vector intercollisional interference" or "vector collision-sequence interference" refer to the fact that an internal $\mathrm{H}_{2}$ scalar transition operator is modulated by a vector function of intermolecular displacement. Thus only a $Q$ branch is observed, with the intercollisional dynamics being those which describe the vector property (intermolecular force, to a good approximation) of the external coordinates.

The present work is based on that of paper I, which will henceforth be referred to as paper I. In paper it was assumed that the collisions suffered by a molecule occur at equally spaced times. This drastic Ansatz allowed the use of the apparatus of discrete Fourier transforms. In this present work it is assumed that the collision times of a given molecule are distributed exactly as a Poisson process, which is in fact an excellent approximation to reality (see $[9,10])$. A summary of this work has appeared in [11].

\section{Poisson-Distributed Collision Times}

At sufficiently low densities and for the study of interference phenomena collisions can be assumed to be instantaneous; the dipole moment induced in one atom or molecule by interaction with a bath of dissimilar atoms or molecules can be represented as

$$
\boldsymbol{\mu}(t)=\sum_{k} \boldsymbol{\mu}_{k} \delta\left(t-t_{k}\right) e^{-\imath \omega_{0} t}
$$


where binary collision $k$ occurs at time $t_{k}$ and the dipole moment induced in collision $k$ is $\boldsymbol{\mu}_{k}$. For the vector interference dips in $Q$ branches the quantity $\boldsymbol{\mu}_{j}$ is parallel to and approximately proportional in magnitude to the impulse (integrated force) $\mathbf{f}_{k}$ experienced by a molecule in the collision. Our models will be expressed in terms of these impulses $\mathbf{f}_{k}$. A second assumption is that the velocities before and after a collision are uncorrelated and are Gaussian:

$$
P\left(\ldots, \mathbf{v}_{k}, \mathbf{v}_{k+1}, \mathbf{v}_{k+2}, \ldots\right)=\ldots P\left(\mathbf{v}_{k}\right) P\left(\mathbf{v}_{k+1}\right) P\left(\mathbf{v}_{k+2}\right) \ldots,
$$

where

$$
P(\mathbf{v})= \begin{cases}\frac{1}{2 \pi} e^{-(1 / 2) v^{2}} & \text { in two dimensions } \\ \frac{1}{(2 \pi)^{3 / 2}} e^{-(1 / 2) v^{2}} & \text { in three dimensions. }\end{cases}
$$

Then the persistence of velocity is zero. This is a fair approximation in some useful cases, such as $\mathrm{H}_{2}-\mathrm{H}_{2}, \mathrm{H}_{2}-\mathrm{He}$, and $\mathrm{HD}-\mathrm{He}$, and a good approximation for systems such as $\mathrm{H}_{2}$ in Ar. It is inappropriate for high-mass atoms or molecules dilute in a fluid of low-mass molecules, such as Ar in $\mathrm{H}_{2}$. Equations (2) and (3) are exact for hard spheres of equal mass, as was first shown by Clerk Maxwell [12].

In general, as stated above, the collision times $t_{k}$ approximate to a Poisson process, and in the present work it will be assumed that they are drawn from a true Poisson process, with frequency $\bar{v}$, whereas in paper I the collisions were assumed to occur at equal intervals.

Equation (1) describes the transition moment for a transition with frequency $\omega_{0}$ in the absence of shifting and broadening mechanisms.

It will be assumed initially that $N$ collisions lie in the finite-time time interval $[0, T)$ such that $0 \leq t_{1} \leq \cdots \leq$ $t_{N-1} \leq t_{N} \leq T$. The time $T$ is of course a random variable if $N$ is fixed, but when $N$ is large the record length $T$ can be taken equal to its expectation value, which will be $N / \bar{\nu}$. The Fourier transform of $\boldsymbol{\mu}(t)$ is

$$
\begin{aligned}
\mathbf{a}(\omega) & =\int_{-\infty}^{\infty} e^{i \omega t} \boldsymbol{\mu}(t) d t \\
& =\sum_{k} \boldsymbol{\mu}_{k} e^{i \tilde{\omega} t_{k}},
\end{aligned}
$$

where $\widetilde{\omega} \equiv \omega-\omega_{0}$. The unaveraged periodogram is given by

$$
\frac{1}{T}|\mathbf{a}(\omega)|^{2}=\frac{1}{T} \sum_{k} \sum_{k^{\prime}} \boldsymbol{\mu}_{k} \cdot \boldsymbol{\mu}_{k^{\prime}}^{*} e^{i \widetilde{\omega}\left(t_{k}-t_{k^{\prime}}\right)},
$$

whence the spectrum itself is given by

$$
\begin{aligned}
S(\omega)= & \lim _{T \rightarrow \infty} \frac{1}{T}\left\langle|\mathbf{a}(\omega)|^{2}\right\rangle \\
= & \bar{\nu}\left[\left\langle\boldsymbol{\mu}_{k} \cdot \boldsymbol{\mu}_{k}^{*}\right\rangle+2 \mathfrak{R e}\left\langle\boldsymbol{\mu}_{k} \cdot \boldsymbol{\mu}_{k+1}^{*} e^{i \tilde{\omega}\left(t_{k}-t_{k+1}\right)}\right\rangle\right. \\
& \left.+2 \Re \mathfrak{R e}\left\langle\boldsymbol{\mu}_{k} \cdot \boldsymbol{\mu}_{k+2}^{*} e^{i \tilde{\omega}\left(t_{k}-t_{k+2}\right)}\right\rangle+\cdots\right] .
\end{aligned}
$$

In the present class of models, as stated above, $\boldsymbol{\mu}_{k}$ is expressed in terms of the impulse $\mathbf{f}_{k}$. Hence, in full generality,

$$
\boldsymbol{\mu}_{k}=\boldsymbol{\mu}_{k}\left(\mathbf{v}_{k}, \mathbf{v}_{k+1}\right)
$$

whence, by (2) and (3),

$$
\left\langle\boldsymbol{\mu}_{k} \cdot \boldsymbol{\mu}_{k+p}\right\rangle=\left\langle\boldsymbol{\mu}_{k}\right\rangle^{2}=0 \text { for } p \geq 2
$$

Then (6) for the spectrum becomes

$$
\frac{S(\omega)}{\bar{\nu}}=\left\langle\boldsymbol{\mu}_{k} \cdot \boldsymbol{\mu}_{k}^{*}\right\rangle+2 \mathfrak{R e}\left\langle\boldsymbol{\mu}_{k} \cdot \boldsymbol{\mu}_{k+1}^{*} e^{i \widetilde{\omega}\left(t_{k}-t_{k+1}\right)}\right\rangle .
$$

A principal assumption of the present model, and the feature in which it differs from the class of models discussed in paper I, is that the intervals $\Delta_{k} \equiv t_{k+1}-t_{k}, k=1,2, \ldots, N-$ 1 between collisions are independent of the velocities of the particle, that is, the collision times $t_{k}, \ldots, t_{k^{\prime}}, \ldots$ are random variables which constitute a Poisson process. Poissondistributed collision times are a good approximation for real gases [9], even at high densities [10], though not exact.

If the random variables $\ldots t_{k}, \ldots, t_{k^{\prime}}, \ldots$ form a Poisson process, then the intervals $\Delta_{i}$ are exponentially distributed [13]:

$$
P\left(\Delta_{i}\right)=\bar{\nu} e^{-\bar{v} \Delta_{i}}
$$

The intervals $\left[0, t_{1}\right)$ and $\left[t_{N}, T\right)$ of durations $\Delta_{0}$ and $\Delta_{N}$, respectively, also follow the distribution law (11), this constitutes a well-known "paradox" in the theory of Poisson processes.

From (10) it follows immediately that

$$
\left\langle e^{i \widetilde{\omega}\left(t_{k}-t_{k+1}\right)}\right\rangle=\left\langle e^{-i \tilde{\omega} \Delta_{i}}\right\rangle=\bar{\nu} \int_{0}^{\infty} e^{-(\bar{\nu}+\imath \widetilde{\omega}) \Delta} d \Delta=\frac{\bar{\nu}}{\bar{\nu}+\imath \widetilde{\omega}} .
$$

Then the spectrum is, from (9), given by

$$
\frac{S(\omega)}{\bar{\nu}}=\left\langle\boldsymbol{\mu}_{k} \cdot \boldsymbol{\mu}_{k}^{*}\right\rangle+2 \mathfrak{R e}\left\{\left\langle\boldsymbol{\mu}_{k} \cdot \boldsymbol{\mu}_{k+1}^{*}\right\rangle \frac{\bar{\nu}}{\bar{\nu}+\imath \widetilde{\omega}}\right\} .
$$

The dipole moment or transition moment induced in a collision is roughly but not exactly proportional to the intermolecular force; the overlap parts differ in range by about $25 \%$. For purposes of calculating the intercollisional interference the integrated induced dipole moment $\boldsymbol{\mu}_{k}$ can be taken parallel to the intermolecular force $\mathbf{f}_{k}$, but with magnitude proportional to some nonlinear function of the magnitude of the intermolecular force; specifically, we consider

$$
\boldsymbol{\mu}_{k}=\mathbf{f}_{k}\left(1+\alpha f_{k}^{\beta}\right)=\left(1+\alpha\left|\mathbf{v}_{k+1}-\mathbf{v}_{k}\right|^{\beta}\right)\left(\mathbf{v}_{k+1}-\mathbf{v}_{k}\right),
$$

where $\alpha$ and $\beta$ are constants, which will not in general be integerial. With this model for $\boldsymbol{\mu}_{k}$, the calculation of

$$
\begin{aligned}
& \max S=\lim _{\omega \rightarrow \infty} S(\omega)=\left\langle\boldsymbol{\mu}_{k} \cdot \boldsymbol{\mu}_{k}\right\rangle, \\
& \min S=S(0)=\left\langle\boldsymbol{\mu}_{k} \cdot \boldsymbol{\mu}_{k}\right\rangle+2\left\langle\boldsymbol{\mu}_{k} \cdot \boldsymbol{\mu}_{k+1}\right\rangle
\end{aligned}
$$

is exactly the same as in paper I. 
2.1. Two Dimensions. For two dimensions it was found in paper I that

$$
\begin{gathered}
\left\langle\boldsymbol{\mu}_{k} \cdot \boldsymbol{\mu}_{k}\right\rangle=4+\alpha 2^{\beta+3} \Gamma\left(\frac{\beta}{2}+2\right)+\alpha^{2} 2^{2 \beta+2} \Gamma(\beta+2), \\
\left\langle\boldsymbol{\mu}_{k} \cdot \boldsymbol{\mu}_{k+1}\right\rangle=-2-2 \alpha \mathcal{g}(\beta+1,1)-\alpha^{2} \boldsymbol{g}(\beta+1, \beta+1),
\end{gathered}
$$

where

$$
\begin{aligned}
\mathscr{g}\left(\nu, \nu^{\prime}\right) \equiv & \left\langle\left(\mathbf{v}_{k}-\mathbf{v}_{k-1}\right) \cdot\left(\mathbf{v}_{k+1}-\mathbf{v}_{k}\right)\left|\mathbf{v}_{k}-\mathbf{v}_{k-1}\right|^{\nu-1}\right. \\
& \left.\times\left|\mathbf{v}_{k+1}-\mathbf{v}_{k}\right|^{\nu^{\prime}-1}\right\rangle \\
= & \frac{3^{\left(\nu+\nu^{\prime}\right) / 2+1} \Gamma(\nu / 2+3 / 2) \Gamma\left(\nu^{\prime} / 2+3 / 2\right)}{8} \\
& \times{ }_{2} F_{1}\left(\frac{\nu}{2}+\frac{3}{2}, \frac{\nu^{\prime}}{2}+\frac{3}{2} ; 2 ; \frac{1}{4}\right) .
\end{aligned}
$$

Note that $\nu$ and $\nu^{\prime}$ are exponents, typically fractional, and are not connected in any way with the mean collision frequency $\bar{v}$.

Because

$$
g(\beta+1,1)=2^{\beta+1} \Gamma\left(\frac{\beta}{2}+2\right)
$$

it follows that

$$
\min S=2 \alpha^{2}\left(2^{2 \beta+1} \Gamma(\beta+2)-\mathscr{g}(\beta+1, \beta+1)\right) .
$$

For the power-law model, which is a limiting case for $\alpha \gg$ 1 of (13) such that

$$
\boldsymbol{\mu}_{k}=\mathbf{f}_{k} f_{k}^{\beta}
$$

with $\beta \approx-0.25$ (for which value see [14]), it can be shown that

$$
\min S=2\left(2^{2 \beta+1} \Gamma(\beta+2)-\mathcal{g}(\beta+1, \beta+1)\right)=\mathcal{O}\left(\beta^{2}\right)
$$

while

$$
\max S=2^{2 \beta+2} \Gamma(\beta+2) .
$$

For the case $\beta=-0.25$, we obtain

$$
\left.\frac{\min S}{\max S}\right|_{\beta=-0.25}=0.0088399 .
$$

2.2. Three Dimensions. The necessary integrals to evaluate $\min S$ and $\max S$ in three dimensions are evaluated in Appendix A. It is found that

$$
\begin{aligned}
\left\langle\boldsymbol{\mu}_{k} \cdot \boldsymbol{\mu}_{k}\right\rangle= & 6+2 \alpha \frac{2^{\beta+3}}{\sqrt{\pi}} \Gamma\left(\frac{\beta}{2}+\frac{5}{2}\right)+\alpha^{2} \frac{2^{2 \beta+3}}{\sqrt{\pi}} \Gamma\left(\beta+\frac{5}{2}\right), \\
\left\langle\boldsymbol{\mu}_{k} \cdot \boldsymbol{\mu}_{k+1}\right\rangle= & -g_{3 D}(1,1)-2 \alpha g_{3 D}(\beta+1,1) \\
& -\alpha^{2} g_{3 D}(\beta+1, \beta+1) \\
= & -3-2 \alpha \frac{2^{\beta+2}}{\sqrt{\pi}} \Gamma\left(\frac{\beta}{2}+\frac{5}{2}\right) \\
& -\alpha^{2} \frac{3^{\beta+3 / 2}}{2 \pi} \Gamma\left(\frac{\beta}{2}+\frac{5}{2}\right)^{2} \\
& \times{ }_{2} F_{1}\left(\frac{\beta}{2}+\frac{5}{2}, \frac{\beta}{2}+\frac{5}{2} ; \frac{5}{2} ; \frac{1}{4}\right) .
\end{aligned}
$$

Then from (14), it follows that

$$
\begin{aligned}
& \min S= \alpha^{2}\left[\frac{2^{2 \beta+3}}{\sqrt{\pi}} \Gamma\left(\beta+\frac{5}{2}\right)-\frac{3^{\beta+3 / 2}}{\pi} \Gamma\left(\frac{\beta}{2}+\frac{5}{2}\right)^{2}\right. \\
&\left.\times{ }_{2} F_{1}\left(\frac{\beta}{2}+\frac{5}{2}, \frac{\beta}{2}+\frac{5}{2} ; \frac{5}{2} ; \frac{1}{4}\right)\right] \\
& \max S=6+\mathcal{O}(\alpha) .
\end{aligned}
$$

The fact that

$$
\frac{\min S}{\max S} \propto \alpha^{2}
$$

is in accord with discussions in $[2,15]$.

For the power-law model given in (19), we have

$$
\min S=0.58 \beta^{2}+\mathcal{O}\left(\beta^{3}\right),
$$

$$
\max S=6+\mathcal{O}(\beta)
$$

and, for $\beta=-0.25$, we obtain

$$
\left.\frac{\min S}{\max S}\right|_{\beta=-0.25}=0.0064980
$$

which may be compared with the two-dimensional value given in (22).

\section{Conclusions}

In this paper, we have extended a class of model developed in paper I for the study of collision-sequence interference effects in collision-induced absorption, to include realistic distributions of collision times. In these models, a single particle is followed. Its collisions are supposed to 
be instantaneous. In paper I the collisions were assumed to occur at equally spaced times, whereas in the present work the collision times are distributed according to a Poisson process. Velocities are supposed to be completely randomized at each collision. It is supposed that the dipole moment $\boldsymbol{\mu}_{k}$ or $\mu_{k}$ induced in a collision is proportional to the integrated intermolecular force $\mathbf{f}_{k}$ or $f_{k}$, respectively, or a power or sum of powers of the integrated intermolecular force.

It is important that the model spectra can be determined analytically, or at worst, reduced to straightforward numerical integrations. The models of paper I, of [11], and of the present work are among the few exactly soluble but nontrivial models found in spectral line-shape studies.

The extension of the induced dipole moment model to dipole moments which are proportional to an arbitrary power of the integrated intermolecular force shows that the interference dip is partially filled in for any disproportionality between induced dipole moment and integrated induced dipole moment. In this paper, the calculation is given for the three-dimensional case. For a realistic value of the power the infilling is slight, being about $0.6 \%$ of spectral maximum for the three-dimensional case.

\section{Appendix}

\section{A. Evaluation of Certain Integrals}

A.1. Evaluation of $\left\langle f^{\nu}\right\rangle$. In three dimensions, the Gaussian distribution of velocities is given by

$$
P(\mathbf{v})=\frac{1}{(2 \pi)^{3 / 2}} e^{-(1 / 2) v^{2}}
$$

whence

$$
\left\langle f^{v}\right\rangle=\frac{1}{8 \pi^{3}} \int d^{3} v d^{3} v^{\prime} e^{-(1 / 2)\left(v^{2}+v^{\prime 2}\right)}\left|\mathbf{v}-\mathbf{v}^{\prime}\right|^{v}
$$

which, setting $\mathbf{u}=\mathbf{v}-\mathbf{v}^{\prime}$ and $\mathbf{U}=(1 / 2)\left(\mathbf{v}+\mathbf{v}^{\prime}\right)$, yields

$$
\begin{aligned}
\left\langle f^{\nu}\right\rangle & =\frac{1}{8 \pi^{3}} \int d^{3} u d^{3} U e^{-(1 / 4) u^{2}-U^{2}} u^{\nu} \\
& =\frac{2}{\pi}\left(\int_{0}^{\infty} d U U^{2} e^{-U^{2}}\right)\left(\int_{0}^{\infty} d u e^{-(1 / 4) u^{2}} u^{\nu+2}\right) \\
& =\frac{2^{v+1}}{\sqrt{\pi}} \Gamma\left(\frac{\nu+3}{2}\right) .
\end{aligned}
$$

Then

$$
\langle f\rangle=\frac{4}{\sqrt{\pi}}=2.2567583, \quad\left\langle f^{2}\right\rangle=6
$$

A.2. Evaluation of $g_{3 D}\left(\nu, v^{\prime}\right)$. For these vectorial cross terms we have

$$
\begin{aligned}
\mathscr{G}_{3 D}\left(\nu, v^{\prime}\right)= & -\frac{1}{(2 \pi)^{9 / 2}} \int d^{3} v d^{3} v^{\prime} d^{3} v^{\prime \prime} e^{-(1 / 2)\left(v^{2}+v^{\prime 2}+v^{\prime \prime 2}\right)} \\
& \times\left|\mathbf{v}-\mathbf{v}^{\prime}\right|^{\nu-1}\left|\mathbf{v}^{\prime}-\mathbf{v}^{\prime \prime}\right|^{\nu^{\prime}-1}\left(\mathbf{v}-\mathbf{v}^{\prime}\right) \cdot\left(\mathbf{v}^{\prime}-\mathbf{v}^{\prime \prime}\right) .
\end{aligned}
$$

We set $\mathbf{u}=\mathbf{v}-\mathbf{v}^{\prime}$ and $\mathbf{u}^{\prime}=\mathbf{v}^{\prime}-\mathbf{v}^{\prime \prime}$ and $\mathbf{U}=(1 / 2)\left(\mathbf{v}+\mathbf{v}^{\prime}+\mathbf{v}^{\prime \prime}\right)$ so that

$$
\begin{gathered}
\left(\begin{array}{c}
\mathbf{v} \\
\mathbf{v}^{\prime} \\
\mathbf{v}^{\prime \prime}
\end{array}\right)=\left(\begin{array}{c}
\frac{2}{3} \mathbf{u}+\frac{1}{3} \mathbf{u}^{\prime}+\frac{2}{3} \mathbf{U} \\
-\frac{1}{3} \mathbf{u}+\frac{1}{3} \mathbf{u}^{\prime}+\frac{2}{3} \mathbf{U} \\
-\frac{1}{3} \mathbf{u}-\frac{2}{3} \mathbf{u}^{\prime}+\frac{2}{3} \mathbf{U}
\end{array}\right), \\
\frac{1}{2}\left(v^{2}+v^{\prime 2}+v^{\prime \prime 2}\right)=\frac{1}{3} u^{2}+\frac{1}{3} \mathbf{u} \cdot \mathbf{u}^{\prime}+\frac{1}{3} u^{\prime 2}+\frac{2}{3} U^{2} .
\end{gathered}
$$

The Jacobian of the transformation $u_{x}, u_{x}^{\prime}, U_{x} \leftarrow v_{x}, v_{x}^{\prime}, v_{x}^{\prime \prime}$ is

$$
\left|\begin{array}{ccc}
\frac{2}{3} & \frac{1}{3} & \frac{2}{3} \\
-\frac{1}{3} & \frac{1}{3} & \frac{2}{3} \\
-\frac{1}{3} & -\frac{2}{3} & \frac{2}{3}
\end{array}\right|=\frac{2}{3}
$$

Then

$$
\begin{aligned}
g_{3 D}\left(\nu, v^{\prime}\right) & -\frac{1}{54 \sqrt{2} \pi^{9 / 2}} \int d^{3} u d^{3} u^{\prime} d^{3} U \\
& \times e^{-\left((1 / 3) u^{2}+(1 / 3) \mathbf{u} \cdot \mathbf{u}^{\prime}+(1 / 3) u^{\prime 2}+(2 / 3) U^{2}\right)} \\
& \times u^{\nu}\left(u^{\prime}\right)^{\nu^{\prime}} \cos \vartheta \\
= & -\frac{3^{\left(3+v+\nu^{\prime}\right) / 2}}{\pi} \int_{0}^{\infty} d u \int_{0}^{\infty} d u^{\prime} \\
& \times \int_{0}^{\pi} d \vartheta \sin \vartheta u^{\nu+2}\left(u^{\prime}\right)^{\nu^{\prime}+2} e^{-\left(u^{2}+u u^{\prime} \cos \vartheta+u^{\prime 2}\right)} \cos \vartheta \\
= & \frac{2 \times 3^{\left(3+v+\nu^{\prime}\right) / 2}}{\pi} \int_{0}^{\infty} d u \\
& \times \int_{0}^{\infty} d u^{\prime} u^{\nu}\left(u^{\prime}\right)^{\nu^{\prime}} e^{-u^{2}-u^{\prime 2}}\left(u u^{\prime} \cosh u u^{\prime}-\sinh u u^{\prime}\right),
\end{aligned}
$$


where $\vartheta=\measuredangle \mathbf{u}, \mathbf{u}^{\prime}$. This gives

$$
\begin{aligned}
g_{3 D}\left(v, v^{\prime}\right)= & \frac{2 \times 3^{\left(3+v+\nu^{\prime}\right) / 2}}{\pi} \sum_{k=0}^{\infty} \frac{1}{(2 k+1) !(2 k+3)} \\
& \times \int_{0}^{\infty} d u \int_{0}^{\infty} d u^{\prime} u^{2 k+3+v}\left(u^{\prime}\right)^{2 k+3+v^{\prime}} e^{-u^{2}-u^{\prime 2}} \\
= & \frac{3^{\left(3+v+v^{\prime}\right) / 2}}{2 \pi} \Gamma\left(2+\frac{v}{2}\right) \Gamma\left(2+\frac{v^{\prime}}{2}\right) \\
& \times \sum_{k=0}^{\infty} \frac{(2+v / 2)_{k}\left(2+v^{\prime} / 2\right)_{k}}{(2 k+1) !(2 k+3)} \\
= & \frac{3^{\left(1+v+v^{\prime}\right) / 2}}{2 \pi} \Gamma\left(2+\frac{v}{2}\right) \Gamma\left(2+\frac{v^{\prime}}{2}\right) \\
& \times \sum_{k=0}^{\infty} \frac{(2+v / 2)_{k}\left(2+v^{\prime} / 2\right)_{k}}{(5 / 2)_{k} k !}\left(\frac{1}{4}\right)^{k} \\
= & \frac{3^{\left(1+v+v^{\prime}\right) / 2}}{2 \pi} \Gamma\left(2+\frac{v}{2}\right) \Gamma\left(2+\frac{v^{\prime}}{2}\right) \\
& \times{ }_{2} F_{1}\left(2+\frac{v}{2}, 2+\frac{v^{\prime}}{2} ; \frac{5}{2} ; \frac{1}{4}\right) .
\end{aligned}
$$

Then

$$
\begin{aligned}
\mathscr{g}_{3 D}(1,1) & =3 \\
\mathscr{g}_{3 D}(\beta+1,1) & =\frac{2^{\beta+2}}{\sqrt{\pi}} \Gamma\left(\frac{5+\beta}{2}\right), \\
\mathscr{g}_{3 D}(\beta+1, \beta+1)= & \frac{3^{3 / 2+\beta}}{2 \pi} \Gamma\left(\frac{5+\beta}{2}\right)^{2} \\
& \times{ }_{2} F_{1}\left(\frac{5+\beta}{2}, \frac{5+\beta}{2} ; \frac{5}{2} ; \frac{1}{4}\right) .
\end{aligned}
$$

\section{Acknowledgments}

The author thanks the Department of Physics of the Pennsylvania State University for its hospitality in 1999, in 2000, in 2005, and in 2008; he gratefully acknowledges many useful discussions on collision-induced absorption with Roger Herman; and he thanks Eugene Oks for providing the opportunity to develop this paper. The support from the Natural Sciences and Engineering Research Council of Canada is acknowledged.

\section{References}

[1] L. Frommhold, Collision-Induced Absorption in Gases, Cambridge University Press, Cambridge, UK, 1993.

[2] J. C. Lewis, "Intercollisional interference-theory and experiment," in Phenomena Induced by Intermolecular Interactions, G. Birnbaum, Ed., pp. 215-257, Plenum Press, New York, NY, USA, 1985.
[3] R. M. Herman, "Analysis of the R1(J)- and P1(J)-branch absorption spectrum of HD-rare-gas mixtures: an example of positive intercollisional interference," Physical Review Letters, vol. 42, no. 18, pp. 1206-1209, 1979.

[4] R. M. Herman, R. H. Tipping, and J. D. Poll, "Shape of the R and $\mathrm{P}$ lines in the fundamental band of gaseous HD," Physical Review A, vol. 20, no. 5, pp. 2006-2012, 1979.

[5] R. M. Herman, in Proceedings of the 14thConference on Spectral Line Shapes (ICSLS '99), R. M. Herman, Ed., AIP Conference Proceedings no. 467, pp. 552-555, American Institute of Physics, Melville, NY, USA, 1999.

[6] J. B. Nelson and G. C. Tabisz, "Intracollisional interference in the pure rotational spectrum of HD: determination of the permanent electric dipole moment," Physical Review A, vol. 28, no. 4, pp. 2157-2161, 1983.

[7] A. R. W. McKellar, J. W. C. Johns, W. Majewski, and N. H. Rich, "Interference effects in the spectrum of HD: III. The pure rotational band at $77 \mathrm{~K}$ for HD and HD-Ne mixtures," Canadian Journal of Physics, vol. 62, no. 12, pp. 1673-1679, 1984.

[8] J. C. Lewis, "Elementary statistical models for collisionsequence interference effects," Physical Review A, vol. 77, no. 6, Article ID 062702, 2008.

[9] S. Chapman and T. G. Cowling, The Mathematical Theory of Non-Uniform Gases, Cambridge University Press, Cambridge, UK, 2nd edition, 1964.

[10] J. C. Lewis, "Distributions of collision times for rough disks and for a two-dimensional lorentz gas with non-overlapping fixed scatterers," Chemical Physics Letters, vol. 76, no. 1, pp. 96-100, 1980.

[11] J. C. Lewis and R. M. Herman, "A statistical model for scalar collision-sequence interference," in Proceedings of the 19th Conference on Spectral Line Shapes (ICSLS '08), M. A. Gigoros and M. Á. González, Eds., AIP Conference Proceedings no. 1058, pp. 122-124, American Institute of Physics, Melville, NY, USA, 2008.

[12] J. C. Maxwell, "Illustrations of the dynamical theory of gases," Philosophical Magazine, vol. 19, pp. 19-32, 1860.

[13] W. Feller, An Introduction to Probability Theory and Its Applications, John Wiley \& Sons, New York, NY, USA, 3rd edition, 1968.

[14] R. M. Herman and J. C. Lewis, "Theory of the fundamental vibration-rotation-translation spectrum of $\mathrm{H}_{2}$ in a $\mathrm{C}_{60}$ lattice," Physical Review B, vol. 73, no. 15, Article ID 155408, pp. 1-14, 2006.

[15] J. C. Lewis, "Theory of intercollisional interference effects-II: induced absorption in a real gas," Canadian Journal of Physics, vol. 50, no. 22, pp. 2881-2901, 1972. 


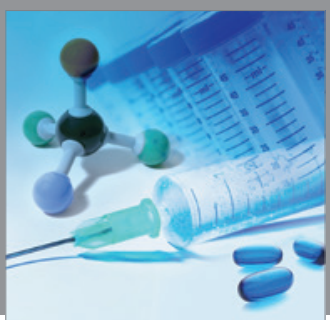

International Journal of

Medicinal Chemistry

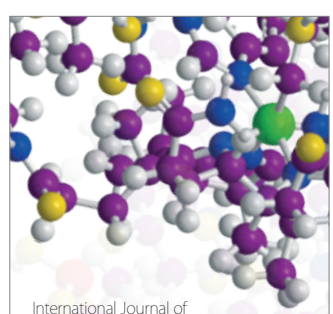

Carbohydrate Chemistry

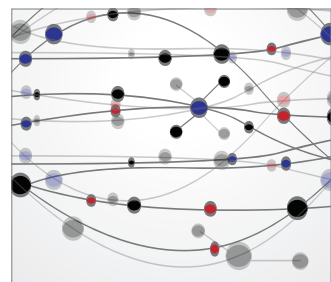

The Scientific World Journal
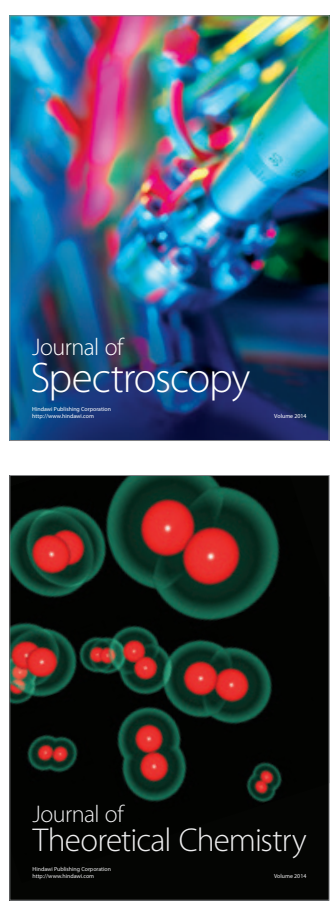
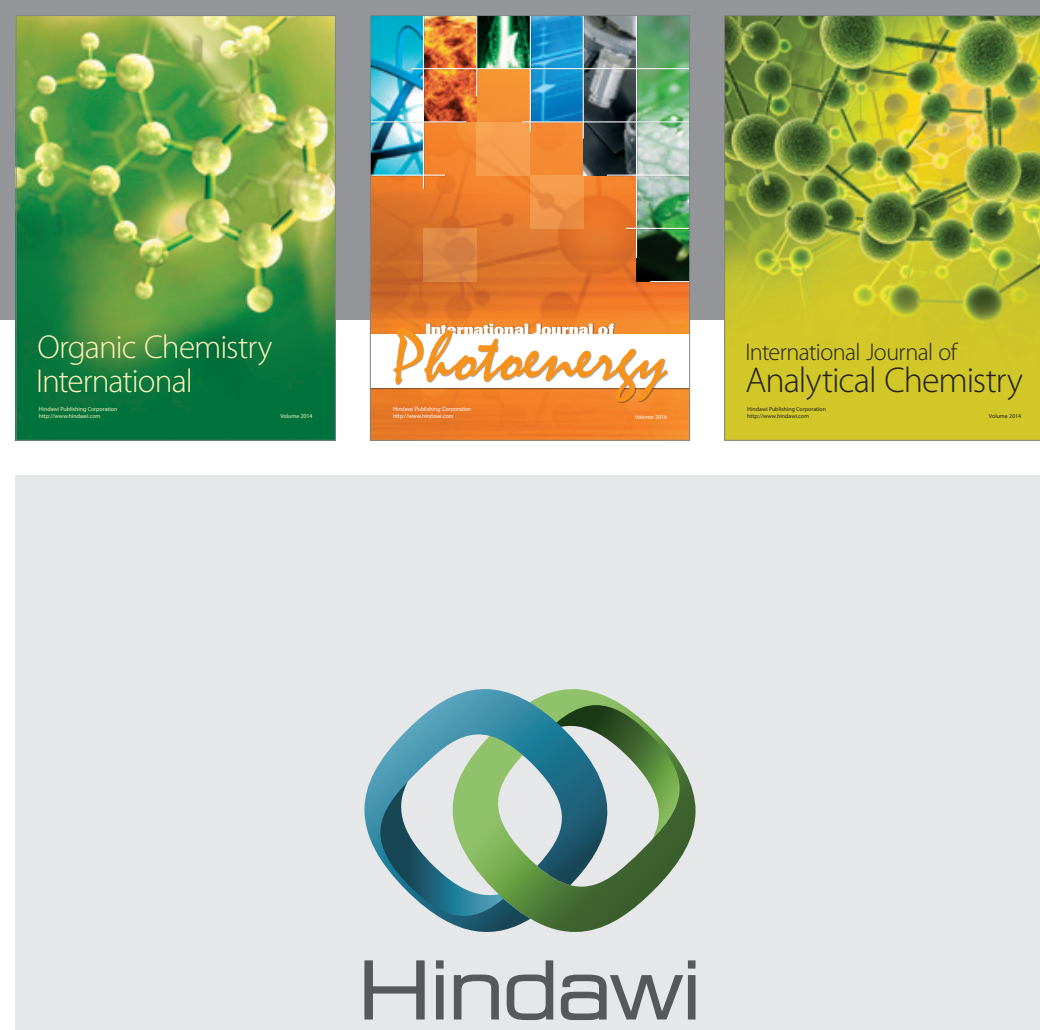

Submit your manuscripts at

http://www.hindawi.com
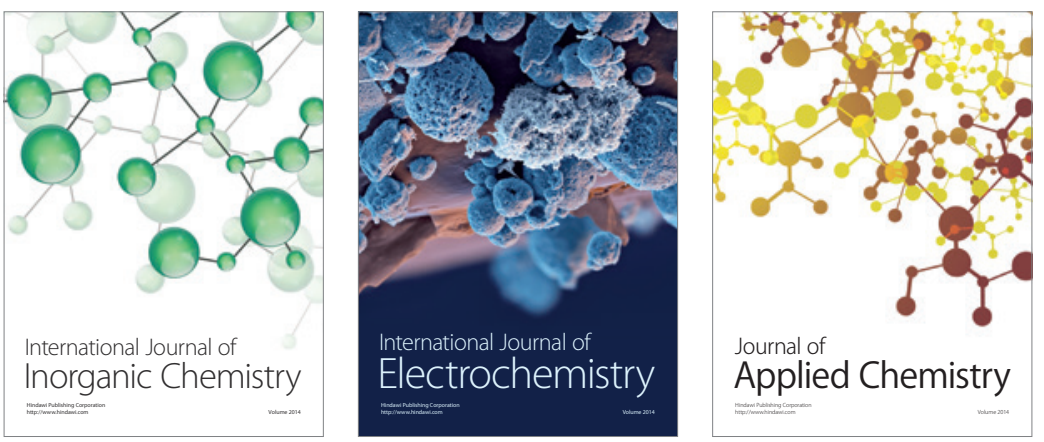

Journal of

Applied Chemistry
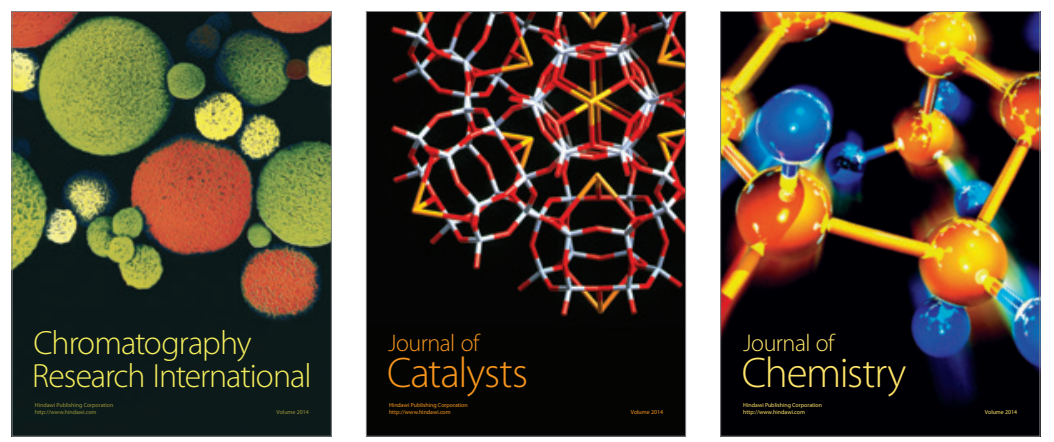
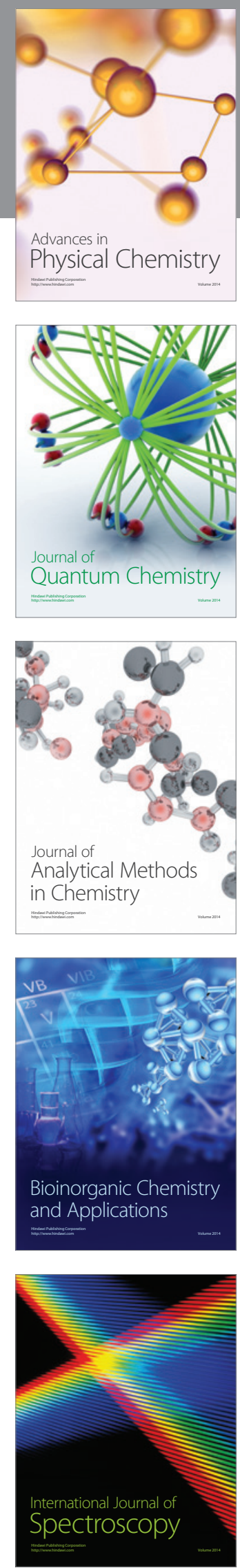